\title{
Culturally Competent Commissioning; Meeting the Needs of Canada's Diverse Communities: The Road Map to a Culturally Competent Mental Health System for All
}

\author{
Tiwalola Foluke Kolapo \\ Middlesex University
}

\begin{abstract}
Cultural competence has proven to be a very efficient tool in reducing healthcare disparities and improving healthcare experiences, compliance with therapy, and reducing incidents of misdiagnosis. This effect is because professionals are recognizing the value and significance of including the person in need of services in their assessment and decision making. While this rationale has also long been considered part of good practice among healthcare professionals (providers) within the mental health arena and nursing care and the success of its use has been reported widely in the provider and insurance arena, the notion seems to have escaped the commissioning arena. Commissioners are responsible for specifying, procuring, and monitoring services and are missing out on the value of completing culturally competent needs assessments for their localities. Synonymous with cultural competence is "person-centred care." In recent times, cultural competence has contributed much to the commissioning of dementia services in a bid to improve and promote person-centred care. It could be argued that there is no person-centred care without cultural competence, which, in simplistic terms, can be defined as care that is undertaken in partnership with the recipient and is of value and significance to the recipient. Culturally competent commissioning and provision of care is therefore to be recommended as capable of addressing quality issues and the problematic variation in services available.
\end{abstract}

Keywords: cultural competence, person-centred care, commissioning, cultural awareness and sensitivities.

Tiwalola F. Kolapo, School of Health and Education, Middlesex University, London, UK.

This manuscript is partly based on a study carried out in conjunction with the completion of a doctorate degree that focused on the culturally competent commissioning of dementia services.

Correspondence concerning this article should be addressed to Tiwalola F. Kolapo, School of Health and Education, Middlesex University, The Burroughs, Hendon, London NW4 4BT, UK. Email: Tk452live@mdx.ac.uk 


\section{RESUMÉ}

Il est démontré que la compétence culturelle est un outil très efficace qui permet de réduire les disparités en matière de soins de santé, d'améliorer la qualité des soins, d'accroître l'observance thérapeutique de de réduire les erreurs de diagnostic. Les professionnels de la santé reconnaissent en effet l'importance d'évaluer les soins et les traitements qu'ils offrent en tenant compte des personnes auxquelles ils sont destinés. Si cela est d'ailleurs depuis longtemps considéré comme faisant partie des meilleures pratiques par les prestataires de soins de santé mentale et infirmiers, et que l'efficacité de cette pratique est largement reconnue par les prestataires de soins et les assureurs, cette notion semble échapper aux commissaires cliniques. Ces derniers, qui doivent définir, obtenir et surveiller les services de santé semblent négliger l'importance de cet aspect. La compétence culturelle est associée à ce que l'on définit maintenant comme les soins axés sur la personne, et depuis quelque temps, elle a beaucoup contribué à un meilleur commissioning de soins de la démence. En d'autres mots, il est impossible d'offrir des soins axés sur la personne sans que la compétence culturelle n'entre en ligne de compte, puisque, en simplifiant un peu, pour que des soins soient axés sur les personnes qui en ont besoin, il faut que l'évaluation de ces soins soit faite avec ces personnes afin qu'ils aient un sens et de la valeur pour elles. Le commissioning et la prestation de soins associés à la compétence culturelle peuvent donc contribuer à améliorer la qualité des soins et à réduire la disparité dans l'offre de soins.

Mots clés : compétence culturelle, soins axés sur la personne, commissioning, sensibilisation aux questions culturelles.

Culturally competent commissioning as proposed here is a reconceptualized framework capable of transforming the delivery of Mental Health (MH) services for a diverse Canadian population. The focus of improvement is on commissioning rather than on delivery. The recommendations do not reinvent the wheel but accept that the Mental Health Commission of Canada (MHCC; "the Commission") has done initial work on culturally competent practice focusing on reducing disparities and risk factors. Furthermore, there has been work by the Commission on improving access to mental health services and strengthening responses to the needs of diverse communities. The Commission is challenged to develop robust key performance indicators, improve knowledge translation from population-based studies, use quality measurement processes, and establish a culturally competent workforce (leadership). The challenge is related to adopting measurements of quality and improvement relevant to Cultural Competence in Commissioning (CCC) - a key component of person-centred care (PCC) in the delivery of support, treatment, and care therapies. There must be recognition of culturally competent commissioning as the starting point for delivering optimal care. The latter cannot be achieved without the former.

\section{THE ISSUES}

In an era of multiculturalism, professionals are experiencing new levels of complexity in service delivery. Issues include reports of poor treatment, low therapy outcomes and compliance, high attrition rates, and poor accessibility due to low health literacy rates. Service users are in turn contending with poor services, discrimination (cultural bias), fragmented care due to badly designed care pathways, and long waiting lists. These are issues that require significant changes in policy and practice and workforce reconfiguration. 
Cultural competency can contend with high levels of mobile groups crossing borders and also meet the needs of indigenes. This was the case when parts of the UK stepped into cultural issues relating to Khat misuse in the Somali community and female genital mutilation practices associated with communities in the diaspora.

Canada, like the rest of the western world, is experiencing higher demands for global health and social care services that meet World Health Organization and human rights standards; it is therefore time to apply the ethics of transcultural care. Madeline Leininger (1995) describes transcultural care as

a formal area of study and practice focused on comparative holistic culture, care, health and illness patterns of people, with respect to differences and similarities in their cultural values, beliefs, and practices with the goal to provide culturally congruent, sensitive, and competent nursing care to people of diverse cultures. (Leininger, 1995)

The diversity and growth in the Canadian population has a dynamic that has resulted in a changing social and political landscape, giving reason to adopt "new" discourses of multiculturalism and cultural competence as an appropriate response to accommodating the health perspectives of the population. The politics of multiculturalism has already seen Canada make a valuable response to discrimination and racism; extending the stance to mental health services is the beginning of cultural awareness on the journey to cultural proficiency. Considering a robust response to the range of cultural needs fulfils the requirements of cultural sensitivity. Cultural awareness and sensitivity are two of four or five constructs associated with the process of cultural competence.

Cultural competence is now a core requirement for mental health professionals working with groups; however, experts agree that without the right training and proficiency in cultural competence, professionals are ill-prepared to do their jobs (Boyle \& Springer, 2001). This is not just about black minority and ethnic groups, as older people are affected by the changing prevalence of dementia and deserve to be part of an inclusive agenda. The agenda must have the capacity to reduce disparities in $\mathrm{MH}$ services and protect human rights or avoid the violation of civil rights (Pope-Davis, Reynolds, Dings, \& Nielson, 1995). The emphasis is beyond provision of good care but on the consideration of what matters to individuals on a cultural level and on improving the quality of $\mathrm{MH}$ care by respecting and understanding cultural perspectives. Recognizing that such ethical practices reduce stigma and attrition rates, improve accessibility and quality, and increase treatment compliance and outcomes is a good start. A study by DeRosa and Kochurka (2006) showed that providing culturally competent care requires clinicians to adapt their own ethnic and biomedical cultural beliefs, values, and practices to those of their patients. They defined and recommended six steps to meet the cultural needs and expectations of patients from diverse populations:

1. Cultivate attitudes associated with excellent transcultural care.

2. Develop an awareness of the impact culture has on the beliefs, values, and practices of the patient and the healthcare provider.

3. Obtain background information about the patient's culture.

4. Perform a cultural assessment.

5. Plan culturally sensitive care using a "preserve-accommodate-restructure" framework.

6. Avoid defensiveness and recover from cultural mistakes. 
The concept of cultural competence is complex to deconstruct as its existence is attached to culture and diversity and its practice lacks a requirement to respond to every expressed aspect of culture. When assessed needs are about ensuring the individual is able to function on a daily basis, care plans are tailored and monitored accordingly. The need may be of a social care nature to meet daily domestic requirements or of a health need to ensure that health is not compromised (nutrition, mobility, respiration, etc.).

A good example of CCC in practice was the case of a substance misuse commissioning team needing to procure a local Structured Day Care program. A culturally competent needs assessment recognized the need to tailor services to suit the needs of Khat users in the Somali community. (Khat is chewed in large quantities to achieve a high and its use is sometimes responsible for domestic violence and family breakdown.) The service included a Somali drug worker to ensure an understanding of the cultural setting, as well as a womenonly service one day per week to increase attendance and investigate the impact of drug use on families.

Canada experiences similar issues to those in the UK where significant numbers of older people are living longer with long-term conditions and significant numbers report lapses in MH brought on by the complexities of modern living, among other factors. There are incidences of migrants reporting PTSD and other illnesses, and increasing numbers of children and young people needing services relating to autism, self-harm, substance misuse, eating disorders, ADHD, and depression. Responses must be innovative in the ways in which they are geared to sustain health in the young and elderly for longer and to combat the higher prevalences of some diseases. This is a challenge that exceeds higher demands for services, gaps in services, and the availability of skilled professionals and associated costs - it is about enabling self-management, rapid response services, sustaining levels of well-being, and transforming the way care is delivered. It is very much about commissioning culturally appropriate care in the right setting, at the right time, and within the confines of best-value principles.

The recommendation offered herein is reflexivity in the professional commissioning rankings and the development of culturally competent policies that aim to provide holistic person-centred care (PCC). By embracing the relevance of cultural competence, we begin the process of elevating its status as a hallmark of quality.

Mental health services in Canada are implored to consider the UK policy tagged "No Health Without Mental Health" (Department of Health \& Social Care, 2011). In the UK, this strategy was a cross-governmental approach to focusing on promoting mental well-being, reducing stigma, and improving outcomes. It promoted the establishment of peer-led recovery and support for carers (as in the Carers Act of 2015) and focused on physical health, reducing mortality rates, improving the length of time people stay healthy, and increasing rapid access to treatment schemes.

The Canadian response should further ensure a cradle-to-grave system of MH care with increased spending on services that emphasize prevention mirroring the UK policy theme, "Start Well, Live Well, Age Well." This means developing programs of health and well-being, including transformation that cuts across all ages and includes systemic and organizational changes. "Starting Well" should look at prevention in the early years, "Living Well" should focus on tackling smoking, alcohol misuse, poor diet/physical activity, and the continuous procurement of services suited to multicultural communities. This direction recognizes that people are living longer with a reduced quality of life impacted by loneliness, poor physical and mental 
health, and poverty. Designing suitable programs that enhance the well-being and protection of the frail and elderly and providing accessible, rapid, and responsive social and healthcare services meets the requirements for the "Aging Well" element of the strategy. The UK mental health strategy has a focus on physical health and re-enablement, further promoting rapid recovery and independence as a means for avoiding long-term entrenched dependencies on services.

Rethinking community needs (population-based commissioning) is not a new idea; it was part of the advice given via Issues and Options (McKenzie, Hansson, Tuck, Lam, \& Jackson, 2009) and The Case for Diversity (McKenzie, Agic, Tuck, \& Antwi, 2016). It requires MH services to develop culturally competent clinicians who can provide culturally competent interventions by using culturally appropriate assessments that produce compliance with treatment to yield improved outcomes.

Ensuring appropriate engagement and a robust understanding of how to work with black and minority ethnic communities requires the cultural competent training of health professionals and commissioners - $\mathrm{a}$ necessity given evidence that cultural competency training improves attitudes, knowledge, skills, and the behaviours of health professionals and impacts positively on patient satisfaction (Renzaho, Romios, Crock, \& Sønderlund, 2013; Betancourt \& Green, 2010; Beach et al., 2005). The direction taken should be towards the provision of flexible services that are sustainable and transformative. New resources are required as well as the reengineering of current services; these play a role in reducing medical errors (Cohen, Rivara, Marcuse, McPhillips, \& Davis, 2005). The issue requires in-depth stakeholder discussions, pooled budgets, and service reconfiguration. There should be no doubt that it requires a review of commissioning practices, adopting Culturally Competent Commissioning, Culturally Competent Needs Assessments/provision of culturally competent care (person-centred care), and a review of how gaps in services should be met based on the demographics of individual Canadian provinces and communities (not a one-size-fits-all approach).

Western countries must note that the continuous commissioning of services based on traditional practices (commissioning for a mono-cultural society) has meant that services are not as fit for purpose as they can be. Such services cannot serve diverse communities; a change requires multifaceted approaches that target structural and systemic aspects of the health system (Alexander, 2008).

\section{THE COMMISSIONING PROCESS}

Commissioning is the process by which services are procured for public consumption based on assessed needs. The process serves to improve the quality of service provision; therefore, the request is that the MHCC considers culturally competent needs assessment policies. Culturally competent commissioning (CCC) is a sub-strategy for commissioning all $\mathrm{MH}$ and services for the elderly. It takes into consideration changing demographics and the higher disease prevalence of some conditions (post-traumatic stress syndrome, ADHD, dementia, autism, etc.). The process must be robustly innovative, cut across systemic and organizational structures in terms of planning via integrated budgets and teams, and employ fair cultural representations (cultural competent workforce/leadership). The new vision is the commissioning and delivery of services that are person-centred and compliant with the principles of global health care.

Policy makers should strive to procure the best services possible within a mixed economy of care while also ensuring that health or social care is accessible and provided free or at a reasonable cost at the 
point of need. It is also crucial that the journey/referral system (care pathway) to accessing care is made seamless and equitable. The complexity of commissioning in the current economy is acknowledged, as are the efforts of commissioners contending with outdated health policies, insufficient availability of providers, limited health budgets, and a lack of skilled manpower. The shortage of skilled staff in social/health care (psychologists, specialist nurses, general practitioners, drug workers, arrest referral workers, and counsellors) affects the provision of quality $\mathrm{MH}$ services. A typical scenario played out in the strategy set out to handle substance misuse in the UK, referred to as the "Tackling Drugs to Build a Better Britain" era. A lack of skilled manpower affected the quality and availability of services available to those needing support. The system required workers from various backgrounds to work in the criminal justice system.

MH strategies draw their success from the robustness of translating good strategies to commissioning and service delivery, hence strategies must constantly be streamlined to respond to varying factors such as limited budgets, limited availability of skilled workers, and the changing dynamics of multicultural populations. There is still the issue of parity of esteem (spending on mental health compared to other health spending) to contend with alongside ensuring value for money, safeguarding (prevention protocols), innovation, and sustainability. Lessons can be learned from the UK system of collaborative commissioning which is employed to ensure joint commissioning via clusters of clinical commissioning groups along with the use of integrated plans and pooled budgets.

\section{CULTURAL COMPETENCE: A REVISITED LANDSCAPE FOR MENTAL HEALTH COMMISSIONING}

Implementing the strategy of Changing Directions, Changing Lives (Mental Health Commission of Canada, 2012) is about providing services that meet higher levels of complex needs, sustaining well-being, improving lives, and preventing mental health lapses. Modifications in clinical practice and organizational performance (Bhui, Warfa, Edonya, McKenzie, \& Bhugra, 2007) should follow. The Commission should define cultural competence in the Canadian MH context because culturally competent policies must fit the setting (not a one-size-fits-all system). It will also have to define delivery, evaluation (key performance indicators), and a budget.

The UK concept "Start Well, Live Well, and End Well" (a cradle-to-the-grave system of care for all) can be adopted and modified to embrace the needs of indigenes, minority groups, and the elderly. It is about transforming what is available into a global system of care capable of achieving equity in the provision of mental health services and ensuring an appreciation by professionals of the complexity of caring for diverse communities (Brown, Cueto, \& Fee, 2006). Studies (e.g., Bhui et al., 2007) refer to the implementation of an assessment and performance framework for cultural competence and the assessment and implementation of measurable benchmarks for performance management. This direction could lay the foundation of a new landscape.

\section{CULTURAL COMPETENCE IN THEORY}

Cultural competence as a discourse first emerged in America as a response to health disparities. The UK, while not adopting cultural competence as a full response to health inequalities, has embraced the concept of 
Person-Centred Care (PCC). Quality person-centred care takes into account the cultural perspectives of the service user; it is care of a health or social care nature that must ask the question, "What matters to you?" PCC shows concordance between care provider and patient within treatment plans, improves health outcomes, and increases patient satisfaction (Ekman et al., 2011). Like culturally competent care, PCC is challenging in practice, and its quality often falls short when cultural competence is not factored in. It is about the ability of a system to provide care to patients with diverse values, beliefs, and behaviours, including tailoring delivery to meet patients' social, cultural, and linguistic needs (Betancourt, Green, \& Carrillo, 2002).

Reflecting on the nature of the concordance refers to obligations the culturally competent professional should honour by respecting the beliefs, language(s), interpersonal styles, and behaviours of individuals and families receiving services (Denboba, 1998).

Cross, Bazron, Dennis, and Isaacs (1989) describe cultural competence as a set of congruent behaviours, attitudes, and policies that come together in a system, agency, or among professionals and enables that system, agency, or those professions to work effectively in cross-cultural situations.

Campinha-Bacote (1998) defines cultural competence as "the process in which healthcare providers consciously strive to achieve the ability and availability to effectively work within the cultural context of a client, be it an individual, family, or community."

We know that culture evolves on an individual and group basis, therefore the aim is to practice cultural competence on an individual basis by finding out what matters to individuals and translating the information into care plans rather than generalizing what is known of a cultural group. Some models of cultural competence (e.g., that put forth by Papadopoulos, 2006) have four constructs, the first of which is Cultural Awareness; this entails an examination of personal value base and beliefs. These authors contend that this is the nature of the construction of cultural identity as well as serving as an influence on people's health beliefs and practices; these are also viewed as planks for a learning platform. Cultural Knowledge can be gained by meaningful contact with individuals from different cultural backgrounds. This can be about forming an understanding of the problems presented and gaining the confidence to influence treatment and therapy compliance; this is conducive to ensuring better treatment outcomes, reductions in attrition rates, and improved customer satisfaction. The attainment of Cultural Sensitivity is instrumental to the delivery of compassionate care; it is the lens through which the professional must view people in their care (Papadopoulos, Tilki, \& Taylor, 1998). Papadopoulos and colleagues (1998) also contend that unless clients are considered as true partners, culturally sensitive or compassionate care cannot be achieved. Cultural sensitivity gives the service user the assurance that there is no "them and I" power divide, causing the service user to be confident enough to express themselves adequately.

The fourth construct is Cultural Competence; it requires the synthesis and application of previously gained awareness, knowledge, and sensitivity. The practical skills required at this stage include assessment of needs, clinical diagnosis, and other caring skills. This completes the model as described by the authors in Transcultural Care: A Guide for Health Care Professionals (Papadopoulos et al., 1998). I introduce a new construct to clarify the need to translate cultural sensitivity, awareness, and knowledge into practice as part of the culturally competent process. Cultural Translation, a proposed fifth construct, is the process of 
using cultural awareness, sensitivity, and knowledge appropriately until cultural proficiency is achieved, as cultural competence cannot be fully achieved by any one individual.

\section{RECOMMENDATIONS:}

\section{Achieving Cultural Competence in Mental Health Services}

Cultural competence can be described as a vehicle or tool that allows professionals to respond sensitively to the perspectives of all cultures present in populations. A constant in $\mathrm{MH}$ services is the presence of a range of complex needs and diverse cultural perspectives concerning those needs. This presents health professionals with the complexity of responding appropriately. Efforts to understand cultural beliefs/practices and language abilities can reduce threats to life resulting from misdiagnosis or non-compliance to treatment. Professionals should adopt new ways of "seeing" the issues that matter to service users, as opposed to labelling sectors of the community "difficult to engage with." This new lens is critical to culturally competent commissioning and the delivery of compassionate care (culturally competent needs assessments as part of service procurement and the development of care plans). The onus is on healthcare providers to ensure they have the ability and capacity to develop co-participation with their service users. The culturally competent practitioner must:

- Refrain from cultural imposition

- Become culturally aware - gain an appreciation of diversity

- Become culturally sensitive - have a positive attitude toward diversity and culture

- Gain cultural knowledge

- Be culturally translational—put cultural knowledge into practice

\section{2: Responding to Diverse MH Needs in Primary and Secondary Care}

In the UK, the first level of care is provided within primary care; however, it is not unusual for some UK GP surgeries (family practitioners) to provide tier 1 and 2 mental health services because some UK clinical commissioning groups commission super surgeries (one-stop multiple family practitioners) to provide primary care mental health and improved access to psychological therapy as a response to the needs of rural communities unable to access services in urban areas. Significant numbers of adults aged 18 to 35 access the emergency department during manic episodes of bi-polar disorder, substance-misuse-related psychosis, and alcohol-related incidences. The recommendation is for out-of-hours advice and information phone lines and the use of "crash pads" (short-term therapeutic residences) as part of a hospital admission avoidance scheme.

\section{Providing Culturally Competent Compassionate Care}

Papadopoulos (2011) defined cultural competence as the capacity to provide effective and compassionate health care, taking into consideration people's cultural beliefs, behaviours, and needs. It's about doing something about those needs using culturally appropriate and acceptable nursing/healthcare interventions 
which take into consideration both the patients' and the carers' cultural backgrounds as well as the context in which care is to be given. This fits the part of the strategy that aims to "Change Lives." Changing lives is about doing things differently: transforming services to deliver much more than treatment and care. A good example is the development of resources for healthcare teams to deliver culturally competent care. Walsh (2004), offers the idea of designing a diagnosis-based nursing documentation computer system to address the unique challenges present in diverse populations. Not only is the system culturally competent, but it also offers easy access.

In recent times in the UK, a public inquiry (Department of Health, 2014) uncovered failings in professional practice at an acute care hospital; the outcome was a call for a change in culture within the National Health System. The inquiry was chaired by Robert Francis QC, presented to Parliament pursuant to Section 26 of the Inquiries Act of 2005, and ordered to print by the House of Commons in 2013. The fruits are a show of how cross-system action is helping the NHS become more transparent, safer, and more compassionate. The report (Sir Francis Report, 2013) set out new measures to further improve organizational culture within the National Health Service. This should be understood in the context of cultural competence at an organizational level, given that the nature of the failings was organizational, systemic, and individual.

The story that sparked the inquiry was linked to several avoidable deaths and the appalling suffering of many patients within hospital services run by a provider organization. These were said to have been caused by serious failings in the leadership ranks. The provider was said to have "...failed in its ability to listen to patients and staff alike, ignoring the need to correct deficiencies and tackle an insidious negative culture involving the tolerance of poor standards and a disengagement from managerial and leadership responsibilities." A further allegation was that the provider put too much emphasis on attaining national targets while ignoring patient safety and acceptable standards of care, perhaps for the purpose of seeking independent status.

The lesson for commissioners and providers is to ensure that the need to manage budgets does not compromise patient safety. Commissioners should also recognize that within mental health services, service users are not only dealing with issues of temporary or permanent lapses in mental health, but also with the stigma attached to their illness. It is for this reason, among others, that compassionate care is important. Safety and comfort are also of significant importance when dealing with those who are stigmatized, especially since mental health services are often the only place where service users may feel understood and cared for. Often, young service users lack insight into their illness, and patients, as well as their parents, experience isolation and frustration. These issues make it necessary for professionals to exercise a deeper level of understanding when assessing, planning, and delivering care for these service users.

In community mental health teams, the cultural competent needs assessments of gaps in services can assist improvements in quality, and consultation with the community, and service users must use meaningful, culturally competent engagement to ensure that the cultural impact issues are understood and acted upon.

\section{Developing a Culturally Competent workforce}

Workforce-related considerations include language barriers, communication (how and what we communicate), respect, belief systems, behaviours, and social engagement or interactions with colleagues and services users. The ability of the health professional to fully appreciate aspects of a service user's perspective 
on their illness, communicate effectively, offer an appropriate diagnosis, and obtain therapy compliance is at stake if the workforce is not culturally competent. Training should be offered to all staff to enhance culturally compliant skills, and recruitment should consider the concept of equal opportunities to ensure a multicultural workforce. Overall, this recommendation is about having performance measures for cultural competency that staff are trained to comply with to give all service users greater accessibility and improved treatment and care outcomes. This should be broadly applicable to the concerns of all cultural groups. In addition, measures and benchmarks should be specific to the agencies responsible for delivering care. The summary recommendation here is for organizations to adopt diversity management practices.

\section{Identifying Good Practice}

The culturally competent journey is a process not a static event, therefore should $\mathrm{MH}$ organizations embrace the process, there must be recognition of the dynamism of the process. This means ensuring that alongside any emerging consensus on organizational best practice, organizations should embrace an equally responsive effort to determine how cultural responsivity can be measured. To determine the potential of existing organizational assessment instruments as a base for the further development of cultural competence standards, a comprehensive review of the literature on cultural competence, healthcare access, and health disparities should be completed regularly. A study by Bowen (2008), looked at assessing cultural responsiveness within organizations (appropriate strategies have not been comprehensively determined). The study contends that unlike America, Canada has no real standards or legislation relating to best practice. The content of the study assessment instrument was based on (a) emerging trends in the theoretical literature; (b) academic research; (c) the literature on organizational diversification; (d) criteria, standards, and guidelines from National Standards for Culturally and Linguistically Appropriate Services in Health Care; (e) guidelines and standards developed by other organizations and systems; and (f) descriptions of exemplary programs. Following an analysis, Bowen developed an assessment instrument with eight domains and seven dimensions for use in document review:

- general profile of cultural responsiveness within the organization;

- human resources;

- cultural training;

- language access services;

- organizational framework and integration;

- information for clients and communities;

- data collection, evaluation, and research; and

- partnership with the community.

\section{Evaluating Cultural Competence}

Key performance indicators for culturally competent mental health services are not explicitly defined in the literature. Cornelius and colleagues (2004) noted the lack of published, validated evaluations of the concept by service users or health commissioners. To make further progress, the researchers conducted 
a study to develop a consumer-based assessment tool for cultural competence. As part of the exercise, a 20-person panel of service users, practitioners, and administrators was convened as a cultural competency advisory board. The board came up with eight concepts upon which we might base an evaluation of the key performance indicators of cultural competence (Cornelius et al., 2004):

1. The level of language proficiency of commissioned interpreters

2. The level of understanding professionals have of indigenous practices

3. The level of acceptance of cultural differences by professionals

4. The level of awareness by professionals of the cultural settings of patients

5. The level to which professionals show respectful behaviours

6. The level of patient-provider organization interactions

7. The levels of consumer involvement

8. The levels of consumer outreach/interventions; for example, culturally adapted CBT (e.g., tailoring services to meet assessed needs which may be related to language, behaviour, religion, or nutrition. Improving levels of outreach eliminates the labelling of some communities as "difficult to engage with").

\section{SUMMARY}

Stork and colleagues (2001) contend that at the time of their study, despite the requirement to provide culturally competent services in the United States, there are no standardized measureable indicators and that the assessment measures in use do not accurately measure the experiences of service users. Furthermore, it is felt that though there have been a wealth of attempts to implement cultural competence in health care, there is still no real consensus on how to define and operationalize it (Suarez-Balcazar et al., 2011). Others further contend that there is insufficient evidence of the efficacy of cultural competence, hindering its infusion into healthcare organizations (Hayes-Bautista, 2003). Still, there is a need for the MHCC and stakeholders to develop a working definition of cultural competence and consider its relevance to mental health service delivery and commissioning. A study of the literature for culturally competent commissioning found no available definition; however, I offer a first definition for culturally competent commissioning, which is

a vehicle for increasing access to quality services (care and treatment) for populations with diverse values, beliefs, and behaviours, using culturally competent processes, including tailoring service delivery to meet social, cultural, and linguistic needs.-Tiwalola Kolapo

Cultural competence in commissioning and service provision can accomplish the following:

- Provision of person-centred care to all groups

- A better understanding of migrant populations and the variations in perspectives on disease

- A reconfiguration of services to ensure sustainability and transformation on a holistic level, including workforce development

- Reduction in mental health service disparities and discrimination 
- Provision of care on a culturally competent basis, ensuring adequate patient-centred care for all

- Encouraging the use of culturally competent assessment practices at both macro (community) and micro (individual) levels

- Improved treatment compliance and therapy outcomes

- Increased access to a wider range of services

- Negated attitudes towards "difficult to reach communities"

- Improved and better awareness of risk (cultural safety)

- Decreased incidences of misdiagnosis that can often arise from the misinterpretation of religious or cultural beliefs/cultural perspectives of diseases

- Assistance for MH practitioners to have insight when assessing the cognition of non-indigenes and indigenes alike

The UK Public Health Directorate has considered the challenges it needs to overcome by 2020, and Canada has set off in the right direction by developing its mental health strategy. The future of mental health policy is in developing sustainable services as part of a system of care with a robust prevention agenda that has the capacity to serve a growing population living longer, affected by long-term mental health issues, and young children affected by parental mental health issues, depression, substance misuse, autism, and others.

Culturally competent commissioning addresses numerous issues within indigenous, migrant, and ethnic minority communities. Both primary and secondary care services should evolve to accommodate the needs of a diverse populace by reviewing care pathways and mapping services accordingly. It will also be prudent for all health and social care providers and practitioners to recognize and respond to different cultural perspectives of illness, variations in reporting symptoms, and peculiar health-seeking behaviour. This requires changes in professional practice and commissioning, given that professionals reflect and/or impose the norms of society and/or their socioeconomic status on others. There also should be a change in how commissioners measure quality, specify services, and design key performance indicators. Finally, and for the first time, a new construct in the cultural competence process is introduced herein to highlight the requirement for all the constructs of the process to be translated into practice to affect a culturally competent event at any one time. It is not enough to be trained in or to possess cultural sensitivity, awareness, or knowledge; but it is the appropriate application of these skills that translates into a culturally competent event. This should be referred to as cultural translation and defined as the appropriate application of cultural sensitivity, awareness, and knowledge in the design of individual therapies for the provision of person-centred care.

\section{REFERENCES}

Alexander, G. R. (2008). Cultural competence models in nursing. Critical Care Nursing Clinics of North America, 20(4), 415-421.

Beach, M. C., Price, E. G., Gary, T. L., Robinson, K., Gozu, A., Placio, A., \& Cooper, L. (2005). Cultural competence: A systematic review of health care provider educational interventions. Medical Care, 43(4), 356-373.

Betancourt, J., \& Green, A. (2010). Commentary: Linking cultural competence training to improved health outcomes: Perspectives from the field. Academic Medicine, 85(4), 583-585. 
Betancourt, J. R., Green, A. R., \& Carrillo, J. E. (2002). Cultural competence in health care: Emerging frameworks and practical approaches (Vol. 576). New York, NY: Commonwealth Fund, Quality of Care for Underserved Populations.

Bhui, K., Warfa, N., Edonya, P., McKenzie, K., \& Bhugra, D. (2007). Cultural competence in mental health care: A review of model evaluations. BMC Health Services Research, 7, 15.

Bowen, S. (2008). Beyond self-assessment: Assessing organizational cultural responsiveness. Journal of Cultural Diversity, 15(1), 7-15.

Boyle, D. P., \& Springer, A. (2001). Toward a cultural competence measure for social work with specific populations. Journal of Ethnic \& Cultural Diversity in Social Work (9)3-4, 53-71.

Brown, T. M., Cueto, M., \& Fee, E. (2006). The World Health Organization and the transition from "international" to "global" public health. American Journal of Public Health, 96(1): 62-72.

Campinha-Bacote, J. (1998). The process of cultural competence in the delivery of health care services: A culturally competent model of care (3rd ed.). Cincinnati, OH: Transcultural C.A.R.E. Associates.

Cornelius, L. J., Booker, N. C., Arthur, T. E., Reeves, I., \& Morgan, O. (2004). The validity and reliability testing of a consumer-based cultural competency inventory. Research on Social Work Practice 14(3) 201-209.

Cohen, A. L., Rivara, F., Marcuse, E. K., McPhillips, H., \& Davis, R. (2005). Are language barriers associated with serious medical events in hospitalized pediatric patients? Paediatrics, 116(3), 575-579.

Cross, T., Bazron, B., Dennis, K., \& Isaacs, M. (1989). Towards a culturally competent system of care (Vol. 1). Washington, DC: Georgetown University Child Development Center, CASSP Technical Assistance Center.

Denboba, D. L., Bragdon, J. L., Epstein, L. G., Garthright, K., \& Goldman, T. M. (1998). Reducing health disparities through cultural competence. Journal of Health Education, 29(Suppl. 1), S47-S53.

Department of Health. (2014). Hard truths: The journey to putting patients first (Vol. 1 of the Government Response to the Mid Staffordshire NHS Foundation Trust Public Enquiry). Norwich, UK: TSO.

Department of Health \& Social Care. (2011). No health without mental health: A cross-government mental health outcomes strategy for people of all ages. HM Government, UK.

DeRosa, N., \& Kochurka, K. (2006). Implement culturally competent healthcare in your workplace. Nursing Management, 37(10), 18-26.

Ekman, I., Swedberg, K., Taft, C., Lindseth, A., Norberg, A., Brink, E., ... \& Sunnerhagen, K. S. (2011). Person-centered care-Ready for prime time. European Journal of Cardiovascular Nursing, 10(4), 248-251.

Francis, R. (chair). (2013). Report of the Mid Staffordshire NHS Foundation Trust Public Inquiry. London, UK: TSO.

Hayes-Bautista, D. E. (2003). Research on culturally competent healthcare systems: Less sensitivity, more statistics. American Journal of Preventive Medicine, 24(3, Suppl.), 8-9.

Leininger, M. (1995). Teaching transcultural nursing in undergraduate and graduate programs. Journal of Transcultural Nursing, 6(2), 10-26.

McKenzie, K., Agic, B., Tuck, A., \& Antwi, M., for MHCC. (2016). The Case for Diversity. Ottawa, ON: Mental Health Commission of Canada.

McKenzie, K., Hansson, E., Tuck, A., Lam, J., \& Jackson, F. (2009). Improving mental health services for immigrant, refugee, ethno-cultural and racialized groups: Issues and options for service improvement. Calgary, AB: Mental Health Commission of Canada.

Mental Health Commission of Canada. (2012). Changing directions, changing lives: The mental health strategy for Canada. Calgary, AB: Author.

National Institute for Mental Health in England. (2001). Inside outside: Improving mental health services for black and minority ethnic communities in England. Department of Health, UK.

Papadopoulos, I. (2006). The Papadopoulos, Tilki and Taylor model of developing cultural competence. In I. Papadopoulos (Ed.)., Transcultural health and social care: Development of culturally competent practitioners (pp.7-24). Edinburgh: Churchill Livingstone.

Papadopoulos, I. (2011). Courage, compassion and cultural competence. The 13th Anna Reynvaan Lecture, 19th May. Academic Medical Centre, University of Amsterdam, Netherlands.

Papadopoulos I., Tilki M., \& Taylor, G. (1998). Transcultural care: A guide for health care professionals. Salisbury, UK: Quay. 
Pope-Davis, D. B., Reynolds, A. L., Dings, J. G., \& Nielson, D. (1995). Examining multicultural counseling competencies of graduate students in psychology. Professional Psychology: Research and Practice, 26(3), 322-329.

Renzaho, A., Romios, P., Crock, C., \& Sønderlund, A. (2013). The effectiveness of cultural competence programs in ethnic minority patient-centered health care: A systematic review of the literature. International Journal for Quality in Health Care, 25(3), 261-269.

Stork, E., Scholle, S., Greeno, C., Copeland, V. C., \& Kelleher, K. (2001). Monitoring and enforcing cultural competence in Medicaid managed behavioral health care. Mental Health Services Research, 3(3), 169-177.

Suarez-Balcazar, Y., Balcazar, F., Taylor-Ritzler, T., Portillo, N., Rodakowsk, J., Garcia-Ramirez, M., \& Willis, C. (2011). Development and validation of the cultural competence assessment instrument: A factorial analysis. Journal of Rehabilitation, 77(1), 4-13.

Walsh, S. (2004). Formulation of a plan of care for culturally diverse patients. International Journal of Nursing Terminologies and Classifications 15(1), 17-26. 